\title{
DNMT1 and AIM1 Imprinting in human placenta revealed through a genome-wide screen for allele-specific DNA methylation
}

Radhika Das ${ }^{1}$, Yew Kok Lee ${ }^{1}$, Ruslan Strogantsev², Shengnan Jin ${ }^{1}$, Yen Ching Lim', Poh Yong Ng ${ }^{1}$, Xueqin Michelle Lin ${ }^{1}$, Keefe Chng ${ }^{1}$, George SH Yeo ${ }^{3}$, Anne C Ferguson-Smith² and Chunming Ding ${ }^{1 *}$

\begin{abstract}
Background: Genomic imprinting is an epigenetically regulated process wherein genes are expressed in a parentof-origin specific manner. Many imprinted genes were initially identified in mice; some of these were subsequently shown not to be imprinted in humans. Such discrepancy reflects developmental, morphological and physiological differences between mouse and human tissues. This is particularly relevant for the placenta. Study of genomic imprinting thus needs to be carried out in a species and developmental stage-specific manner. We describe here a new strategy to study allele-specific DNA methylation in the human placenta for the discovery of novel imprinted genes.

Results: Using this methodology, we confirmed 16 differentially methylated regions (DMRs) associated with known imprinted genes. We chose 28 genomic regions for further testing and identified two imprinted genes (DNMT1 and AIM1). Both genes showed maternal allele-specific methylation and paternal allele-specific transcription. Imprinted expression for AIM1 was conserved in the cynomolgus macaque placenta, but not in other macaque tissues or in the mouse.

Conclusions: Our study indicates that while there are many genomic regions with allele-specific methylation in tissues like the placenta, only a small sub-set of them are associated with allele-specific transcription, suggesting alternative functions for such genomic regions. Nonetheless, novel tissue-specific imprinted genes remain to be discovered in humans. Their identification may help us better understand embryonic and fetal development.
\end{abstract}

Keywords: Genomic imprinting, Placenta, Next generation sequencing, Differentially Methylated Region (DMR), DNMT1, AIM1

\section{Background}

Genomic imprinting is the epigenetic phenomenon wherein genes are expressed exclusively from one parental allele $[1,2]$. Imprinting has been reported in placental mammals, specifically, in primates, rodents, canines and ruminants. Some of these imprinted genes exhibit speciesspecific and spatial-temporal patterns of imprinted expression $[3,4]$.

Selective inactivation of one parental allele can be achieved by parent-of-origin specific cytosine methylation. Germline-derived heritable differentially methylated regions (gDMRs) are established at the gamete stage [5].

\footnotetext{
* Correspondence: cmding@gmail.com

'Singapore Institute for Clinical Sciences, Agency for Science, Technology and Research (A*STAR), Singapore, Singapore

Full list of author information is available at the end of the article
}

Secondary differentially methylated marks are acquired after fertilization or later in life, and these are known as somatic DMRs or sDMRs [6]. Allele-specific activating or repressive histone modifications have also been implicated in regulating imprinting [7].

Since the discovery of the first imprinted gene in 1991, 73 imprinted genes have been identified in humans http:// igc.otago.ac.nz/home.html while 155 imprinted genes have been reported in mice (http://www.mousebook.org/catalog. php?catalog=imprinting). In recent years, many studies using genome-wide technologies for genomic or epigenomic analyses were performed to identify novel imprinted genes. However, most had mixed success [8-16]. Whole genome and transcriptome sequencing technologies have helped identify only a small number

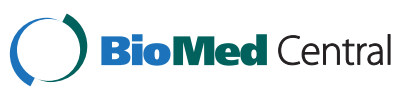

(C) 2013 Das et al.; licensee BioMed Central Ltd. This is an open access article distributed under the terms of the Creative Commons Attribution License (http://creativecommons.org/licenses/by/2.0), which permits unrestricted use, distribution, and reproduction in any medium, provided the original work is properly cited. 
of imprinted transcripts [8,17-22], suggesting that most imprinted genes have already been identified [23] or are tissue-specific and thus needed to be analyzed in specific cell types [8]. Only one study identified a large number of potential imprinted genes in the mouse brain [24,25], but further investigation revealed that most of these may be false positives due to artifacts from the RNA-Seq approach [26], a finding supported by more recent data [27].

Functionally, genomic imprinting is critical for proper placenta and embryo development [28-32]. Conditions such as Intra-Uterine Growth Restriction (IUGR) and pre-eclampsia as well as unsuccessful pregnancies have been correlated with abnormalities in methylation or aberrant expression of imprinted genes in the placenta [33-35]. Surprisingly, very few human or primatespecific placental imprinted genes are known so far, though interesting candidates like RB1 (Table 1), ZNF331 and the microRNA cluster C19MC have been discovered in recent screens [21,36,37]. A comparison between the 73 imprinted genes discovered to date in humans and the 155 reported in mice reveals that majority of this divergence is due to the multiple genes imprinted specifically in the mouse placenta [38,39], although recent data suggests that several genes were wrongly identified as showing imprinted expression in mouse placenta $[18,40,41]$. The imprinting difference is consistent with the biological differences between the less-invasive mouse placenta and its highly invasive human counterpart.
In this study, we used reduced representation bisulfite sequencing (RRBS) to identify partially methylated $\mathrm{CpG}$ islands (CGIs) in the human placental genome. We further identified candidate regions with allele-specific methylation based on calculation of methylation concordance values. We then selected 28 regions for further characterization and identified two novel imprinted genes (DNMT1 and AIM1). Both genes are paternally expressed and methylated specifically on the maternal allele in the human placenta. For AIM1, the differential methylation is conserved in another primate, the cynomolgus macaque but not in the mouse. In conclusion, we have delineated many regions with allele-specific methylation and developed an approach for the identification of human placenta-specific imprinted genes.

\section{Results}

Confirmation of known germline differentially methylated regions using RRBS DNA methylation analysis

Nine human placental samples (five first trimester and four third trimester) were subject to RRBS analysis for DNA methylation (Figure 1). CpG sites sequenced at greater than $10 \times$ coverage were included in the analysis. If our approach was to be used for identifying novel imprinted genes, it should also be able to confirm the known gDMRs. Indeed, CGIs overlapping 14 known human DMRs (known to be gDMRs in mouse) were found to be approximately $50 \%$ methylated (Table 1). The DMRs for the genes MCTS2 and INPP5F_V2 (described

Table 1 Confirmation of known human germline differentially methylated regions

\begin{tabular}{|c|c|c|c|c|}
\hline Gene & Germline DMR locus* & Overlapping UCSC CpG island* & Average methylation & Reference \\
\hline HYMAI/PLAGL1 & chr6:144323557-144324495 & chr6:144328917-144329847 & 0.384 & [42] \\
\hline IGF2R** & chr6:160426265-160427502 & chr6:160426265-160427502 & 0.698 & [43] \\
\hline GRB10 & chr7:50849753-50850871 & chr7:50849753-50850871 & 0.452 & [44] \\
\hline SGCE/PEG10 & chr7:94284859-94286527 & chr7:94284859-94286527 & 0.390 & {$[45]$} \\
\hline MEST & chr7:130130740-130133111 & chr7:130130740-130133111 & 0.499 & [46] \\
\hline H19/IGF2 & chr11:2020180-2022580 & chr11:2019566-2019863 & 0.457 & {$[47]$} \\
\hline KCNQ1 & chr11:2720354-2721827 & chr11:2720411-2722087 & 0.504 & [48] \\
\hline MEG3 & chr14:101272662-101277765 & chr14:101290524-101290868 & 0.334 & [49] \\
\hline SNRPN & chr15:25,199,933-25200342 & chr15:25200036-25201054 & 0.374 & {$[50]$} \\
\hline PEG3 & chr19:57351284-57351995 & chr19:57351284-57351995 & 0.420 & [51] \\
\hline NNAT & chr20:36148604-36150136 & chr20:36148604-36150136 & Not represented & {$[52]$} \\
\hline$\angle 3 M B T L$ & chr20:42143211-42143591 & chr20:42143211-42143591 & 0.479 & [53] \\
\hline GNAS-A & chr20:57464132-57464622 & chr20:57463653-57467739 & 0.191 & [54] \\
\hline GNAS-B & chr20:57426198-57430959 & chr20:57426730-57427047 & 0.494 & [55] \\
\hline$R B 1$ & chr13:48892636-48893857 & chr13:48892636-48893857 & 0.577 & {$[56]$} \\
\hline INPP5F_V2 & chr10:121577530-121578385 & chr10:121577530-121578385 & 0.456 & {$[57]$} \\
\hline MCTS2 & chr20:30135077-30135292 & chr20:30135077-30135292 & 0.633 & {$[57]$} \\
\hline
\end{tabular}

*Based on UCSC genome build hg19.

**Polymorphic imprinting reported in human placenta, but DMR still present. 


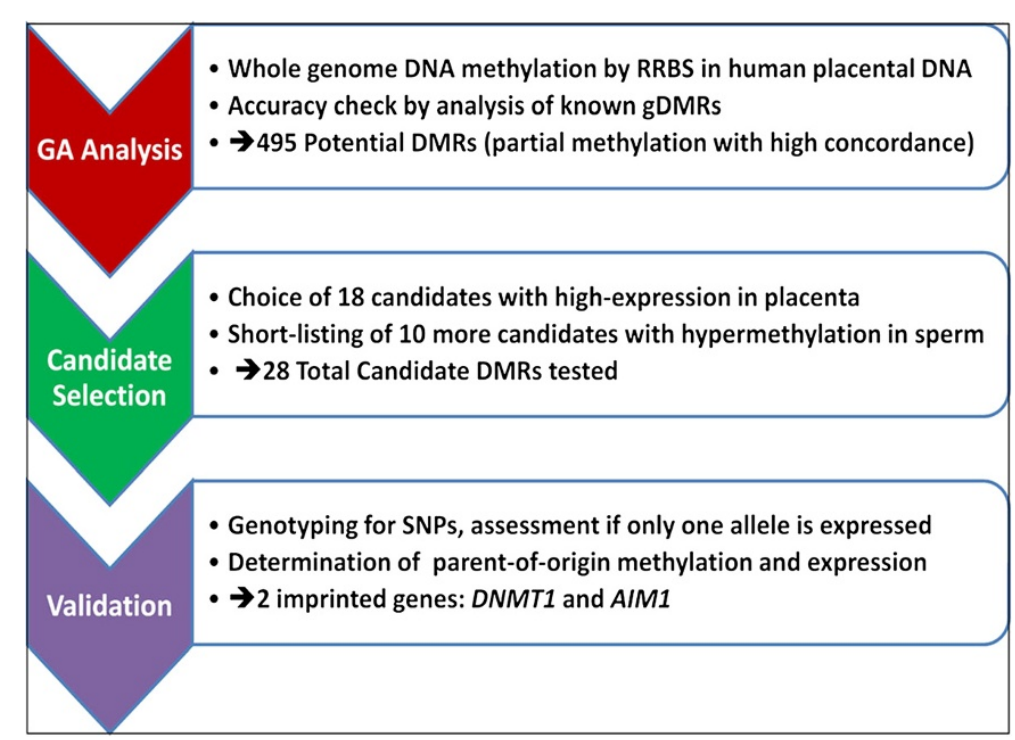

Figure 1 Pipeline for assessment of allele-specific methylation and genomic imprinting in the human placenta. The process involves three main steps - reduced representation bisulfite sequencing of placental samples (red), selection of partially methylated regions with high concordance (green) and individual locus-based validation of the potential DMRs and parental allele-specific expression (violet).

in [57]) were further validated by bisulfite cloning and sequencing and were found to be methylated in an allele-specific manner (Additional file 1: Figure S1). The NNAT promoter was not covered by our sequencing data. For the GNAS locus, the CGI overlapping with the DMR exhibited $19.1 \%$ methylation. However, on analyzing individual $\mathrm{CpG}$ sites within this large CGI, the first half of the CGI was found to be about 50\% methylated.

\section{Allele-specific methylation analysis and selection of potential DMRs}

On calculation of a concordance value (see Methods), the known DMRs were shown to be partially methylated with high concordance (Figure 2A). The mean and median concordance values for the first trimester placentas were $90.9 \%$ and $92.8 \%$ respectively while those for the third trimester placentas were $90.5 \%$ and $93.8 \%$ respectively. However, other partially methylated CGIs (30-70\% methylation) showed a much higher variability in concordance value. We hypothesized that novel DMRs associated with imprinted genes should demonstrate similar methylation patterns to the known DMRs with partial methylation and high concordance.

By choosing partially methylated autosomal CGIs with $>85 \%$ concordance, we identified 953 regions in first trimester placenta and 994 regions in third trimester placenta, 495 of which were shared between the two trimesters (Figure $2 \mathrm{~B}$ ). These regions were located in promoters, gene bodies and inter-genic regions (Figure 2C). Details of these regions are listed in Additional file 2.

Amongst the 495 potential DMRs overlapping between the first trimester and third trimester placenta samples, we chose 28 genomic regions for further validation. The first 18 regions (Table 2) were chosen based on high expression levels of adjacent genes in placenta as ascertained from RNA-seq data (Jin et al. unpublished data). The other 10 regions (Table 3 ) were chosen since they were highly methylated in human spermatozoa samples [58] and also had high expression levels of adjacent genes in placenta. These 10 regions were candidates for paternally methylated gDMRs.

Analysis of allele-specific expression for genes located in the selected regions in human placenta

We chose 28 genes (Tables 2 and 3) associated with the 28 candidate DMRs for analysis of allele-specific expression. Three to four exonic SNPs per gene were analyzed in 28 paired placental DNA and RNA samples. Two genes (DNMT1 and AIM1) showed allele-specific expression. The monoallelic expression profile was not due to biased expression from one specific allele since reciprocal alleles were represented in the sample set (Figure 3D, Figure 4D, Additional file 3: Figure S3A). For DNMT1 (or DNA Methyl Transferase 1), eight heterozygotes (harboring four SNPs in different exons) exhibited a single allele in their cDNA (four examples are shown in Figure 3D). For AIM1 (or Absent in Melanoma 1), there are two alternative transcripts, the long transcript and the short transcript (Additional file 4: Figure S2). Allele-specific expression was observed in 28 individuals with two different SNPs located in exon 1 specific for the long transcript (four examples are shown Figure 4D). However, bi-allelic expression was observed with SNPs located in exon 20 shared by both the long and the short transcript in two individuals 


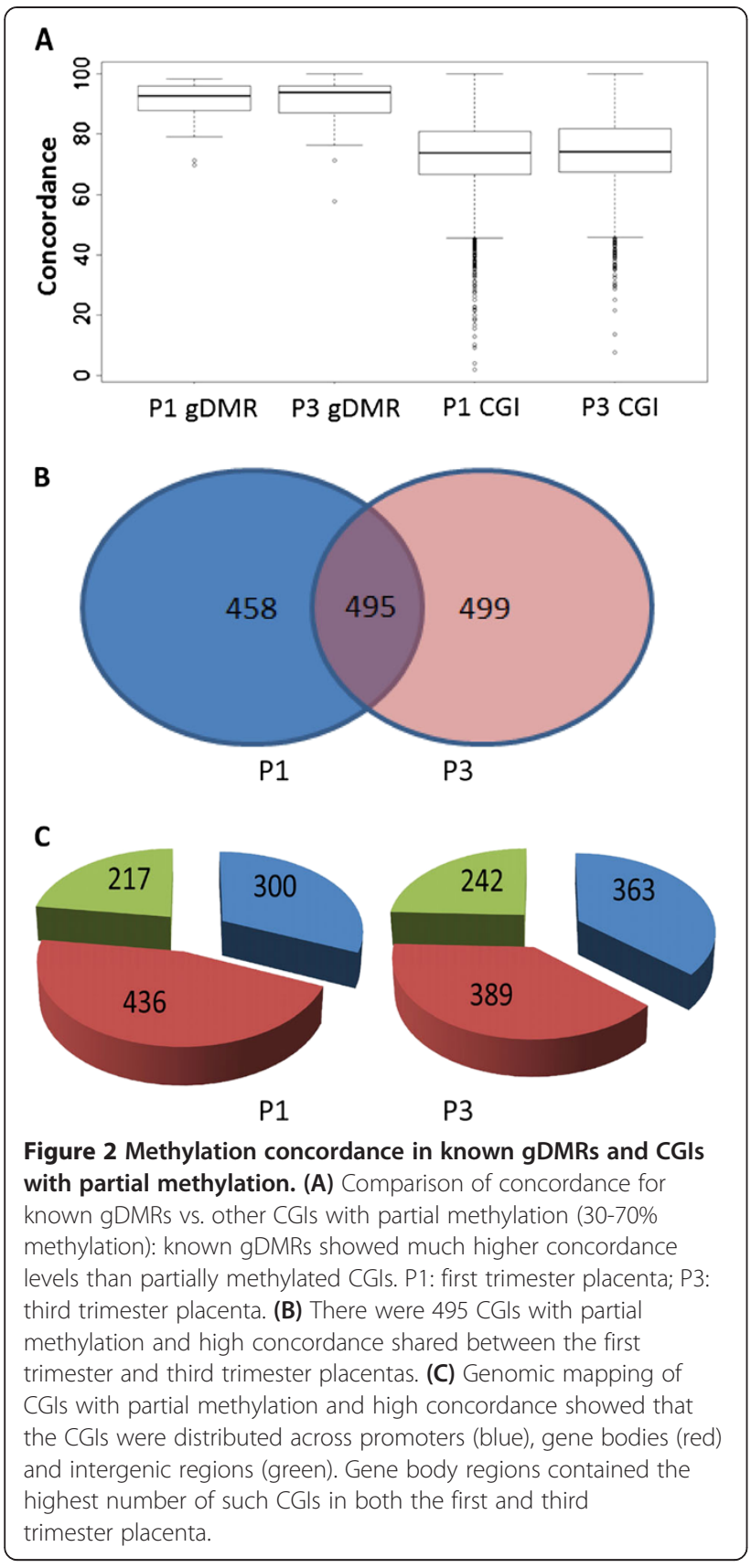

(Additional file 4: Figure S2), indicating that imprinting is limited only to the long transcript of AIM1. The bi-allelic status of the shorter transcript was confirmed by 3' RACE and SNP analysis.

\section{Methylation and Imprinting analyses of DNMT1 in human placenta}

DNMT1 has two alternative transcripts, one expressed in somatic tissues (s-DNMT1) and the other expressed specifically in the oocyte (o-DNMT1). The potential DMR identified at Chromosome 19 CGI 89 is located at the promoter of $s$-DNMT1. We performed bisulfite cloning and sequencing for this region in three human placenta samples to confirm the allele-specific methylation status (one representative example shown in Figure 3B). An individual (fN599) with an informative SNP exhibited methylation of the maternal allele $(\mathrm{T})$ and was unmethylated on the paternal allele (G; Figure $3 \mathrm{C}$ ). The allelespecific methylation profile was confirmed in one more sample (fN134, Additional file 3: Figure S3B). However, parent-of-origin methylation could not be determined for the second sample since the mother was also heterozygous at this locus.

All the eight polymorphic human placenta samples showed monoallelic expression. Informative SNPs were available in four samples where the mothers were homozygous. All four samples showed paternal allele-specific expression of $s$-DNMT1 (Figure 3D).

\section{Methylation and Imprinting analyses of AIM1 in human placenta}

We performed bisulfite cloning and sequencing for four human placental DNA samples for the Chromosome 6 CpG 114 region (located within AIM1). All four samples displayed equal numbers of methylated and unmethylated clones, characteristic of a DMR (one representative example shown in Figure 4B). An individual (fN158) with an informative SNP at the DMR exhibited a methylated maternal allele $(G)$, while the paternal allele $(T)$ was unmethylated (Figure $4 \mathrm{C}$ ). This maternal allelespecific methylation profile was confirmed in one more individual (fN155, Additional file 3: Figure S3C). We also confirmed that the methylation profile was not a SNP effect by profiling a sample (mN158) that was nonpolymorphic at the same locus. This sample still showed the characteristic allele-specific methylation profile (Additional file 3: Figure S3D).

Allele-specific expression was observed in 28 individuals with two different SNPs located in exon 1 specific for the long transcript. Four of the mothers were homozygous at the corresponding SNP loci and thus were informative for parent-of-origin expression analysis. All four placenta samples displayed paternal expression of this gene (Figure 4D).

\section{Methylation and Imprinting analyses of AIM1 in cynomolgus macaque placenta}

The region homologous to human CpG 114 in the macaque was analyzed in the placental DNA of three macaques and shown to have approximately $34 \%$ methylation in each sample (one representative example shown in Figure 5A). Analysis of one macaque (pl36) with a $\mathrm{C} / \mathrm{G}$ polymorphism within the DMR indicated that the expressed allele $(\mathrm{G})$ was completely unmethylated, whereas the non-expressed allele (C) was partially methylated (Figure 5B).

Eleven macaque placental tissues were further analyzed for expression, and four heterozygotes for the first 
Table 2 Partially methylated CpG islands with high concordance

\begin{tabular}{|c|c|c|}
\hline Locus* & CpG island* & Location and annotation \\
\hline chr6: $106959764-106960985$ & CpG 114 & Exon-Intron 1 of AIM1 (melanoma suppressor) \\
\hline chr7: 807336-808261 & CpG 79 & Promoter of HEATR2 alternative transcript \\
\hline chr19:36604359-36606906 & CpG 194 & Overlaps Promoters of TBCB/ POLR21 (polymerase sub-unit gene ) \\
\hline chr19:55992577-55996916 & CpG 321 & Last exon of ZNF628/ promoter of NAT14 (acetyltransferase) \\
\hline chr1:41847264-41849204 & CpG168 & Last exon of FOXO6 \\
\hline chr1:111746337-111747303 & CpG 94 & Promoter of DENN/MADD domain containing 2D \\
\hline chr4:154712073-154712706 & CpG 57 & Downstream of SFRP2 (Wnt singnalling) \\
\hline chr12:22486835-22488666 & Cpg 163 & Promoter of ST8SIA1 (sialyltransferase for ganglioside production) \\
\hline chr13:33001249-33002078 & CpG 93 & Intron 1 of NEDD4 binding protein 2-like 1 isoform 1 \\
\hline chr19:1584445-1585247 & CpG 89 & Exon-intron of MBD3 (Nurd complex subunit: nucleosome remodeling) \\
\hline chr22:29706500_29706710 & CpG 15 & Exon-intron 3-4 of GAS2L1 (similar to Gas2, Actin-associated protein) \\
\hline chr9:36,222,678-36,294,377 & CpG 76 & Promoter of GNE, enzyme for N-Acetyl Neuraminic Acid regulation \\
\hline chr11:497359-511488 & CpG 46 & Exon-intron 2 of RNH1 (placental ribonuclease inhibitor) \\
\hline chr19:10304966-10305864 & CpG 89 & Promoter of DNMT1 (DNA Methyl transferase) \\
\hline chr15:96856299-96875368 & CpG 145 & Upstream of NR2F2 (steroid thyroid family of nuclear receptors) \\
\hline chr2:241496576-241497600 & CpG 96 & Exon-intron of ANKMY1 (Ankyrin repeat and MYND domain containing protein) \\
\hline chr7:127671159-127672853 & CpG 156 & Exon-intron of SND1 (p100 co-activator) \\
\hline chr4:169799086-169799625 & CpG 58 & Exon-intron of PALLD (cytoskeletal protein involved in actin organization) \\
\hline
\end{tabular}

*Based on UCSC genome build hg19.

exon were found. Monoallelic expression was observed in all four samples (Figure 5C). Since parent/offspring matched samples were not available, we were unable to determine the parental origin of the expressed allele in these animals.

\section{Methylation and imprinting analyses of AIM1 in other cynomolgus macaque tissues}

Additional macaque tissues (liver, biceps, kidney, heart, lungs and pancreas) were available for two of the informative individuals, and these were also subject to methylation analysis at the DMR. However, all the tissues were found to be completely unmethylated (Additional file 5: Figure S4). AIM1 was expressed in the heart, kidney and placenta, but showed minimal or no expression in the liver, lung and pancreas by qPCR analysis (Additional file 6: Figure S5). AIM1 was found to be bi-allelically expressed in the kidney and heart in two macaques (example of Macaque 11 is shown in Additional file 6: Figure S5).

\section{Methylation and imprinting analyses of $\operatorname{Aim} 1$ in mice}

Twelve samples from reciprocal crosses of CAST/EiJ and BL6 mice were analyzed for methylation levels in the Aim1 promoter, and in a second region with a potential alternative transcription start site upstream of Aim1. Both regions were hypomethylated in this species (Figure 6A, Additional file 7: Table S3). Aim1 was

Table 3 Partially methylated CpG islands with $100 \%$ methylation in sperm

\begin{tabular}{lll}
\hline Locus* $^{*}$ & CpG island* & Location and annotation \\
\hline chr2:220312699-220314094 & CpG 153 & Internal exon of SPEG (striated muscle protein kinase) \\
chr3:128215213-128216905 & CpG 137 & Upstream of GATA2 (Zinc finger transcription factor) \\
chr5:343450-344535 & CpG 117 & Internal exon-intron AHRR (aryl hydrocarbon receptor repressor) \\
chr5:179740711-179741121 & CpG 43 & Internal exon-intron GFPT2 (controls flux of glucose into hexosamine pathway) \\
chr6:1624186-1625468 & CpG 111 & Promoter of GMDS (catalyzes conversion of GDP-mannose to GDP-4-keto-6-deoxymannose) \\
chr8:145749856-145750410 & CpG 61 & Promoter of LRRC14 (Leucine rich repeat containing protein) \\
chr13:110965775-110966223 & Cpg 43 & Intron of COL4A2 (encodes one sub-unit of Type IV collagen) \\
chr17:46641535-46642110 & CpG 56 & Intron of HOXB3 (homeobox transcription factor needed for development) \\
chr19:36246329-36247982 & CpG 127 & Promoter of HSPB6 (heat shock protein, alpha-crystallin related) \\
chr20:62193967-62198985 & CpG 381 & Internal exon of PRIC285 (part of peroxisome proliferator receptor alpha) \\
\hline
\end{tabular}

*Based on UCSC genome build hg19. 


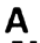

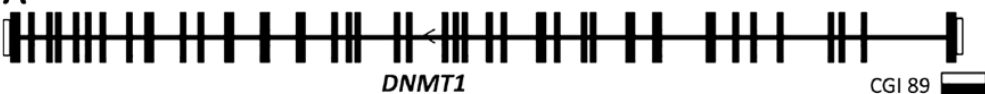

B

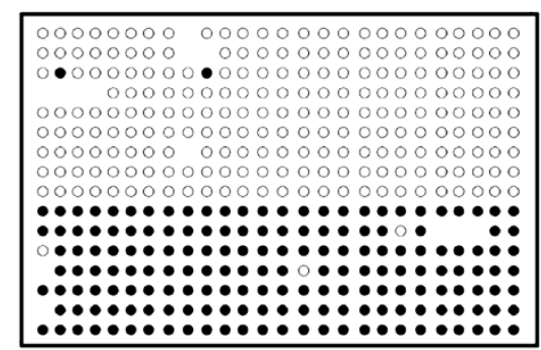

C

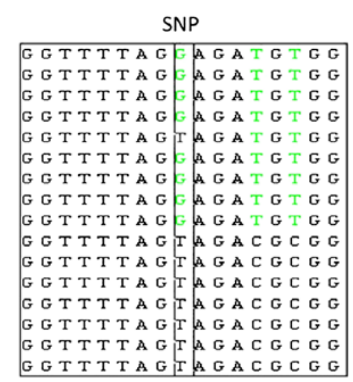

D

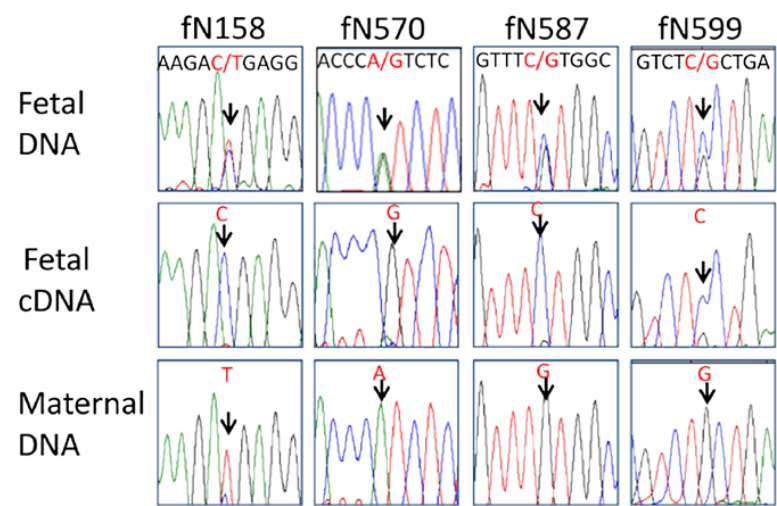

Figure 3 Methylation and expression analyses of DNMT1 in human placenta. (A) CGl 89 is located at the promoter of DNMT1. (B) Bisulfite treatment and cloning confirmed monoallelic methylation. (C) Sequencing of clones from the heterozygous individual fN599 showed that the maternal allele ( $T$ allele) was associated with methylated CpGs, while the paternal allele (G allele) was associated with unmethylated CpGs. (D) DNMT1 was paternally expressed in humans. Arrow depicts the SNP location for each sample. In all four samples, the fetal DNA was heterozygous and the maternal DNA was homozygous. Fetal RNA showed monoallelic expression from the paternal allele.

expressed in the kidney, placenta and heart but showed minimal expression in the liver, brain and lung (Additional file 8: Figure S6) by qPCR analysis. Bi-allelic expression was observed in all tissues (placenta, brain, heart, liver, lung, and kidney) in the reciprocal crosses (Figure 6B).

\section{Discussion}

The human placenta was chosen for our investigation of novel imprinted genes since genomic imprinting is critical for placenta and embryo development. Additionally, morphological and physiological differences are evident between mouse and human placenta, consistent with differences in imprinting between these two species. RRBS was used to quantify DNA methylation at CpG-rich regions, since it allowed us to readily distinguish two different types of partially methylated regions: those with allele-specific methylation which show high concordance; and those that exhibit variable methylation where different $\mathrm{CpGs}$ on the same allele can be methylated or unmethylated [59].
Our DNA methylation data at single base resolution confirmed 16 known DMRs associated with imprinted genes. One known DMR (at the promoter of NNAT) was not confirmed because the genomic region was not analyzed by RRBS. As expected, the known DMRs were partially methylated with high concordance. Thus, we selected 28 candidate DMRs from 495 partially methylated regions with high concordance in both first and third trimester placenta samples for analysis of allelespecific expression of adjacent genes. Subsequently, we confirmed that DNMT1 and AIM1 were maternally methylated and paternally expressed. While we were preparing the manuscript, a similar theoretical model was used to describe allele-specific methylation in the human genome [60]. The authors identified known imprinted DMRs from publically available methylome datasets in predominantly cultured cells. Another related model has also been used to detect allele-specific methylation in the Arabidopsis genome [61]. 

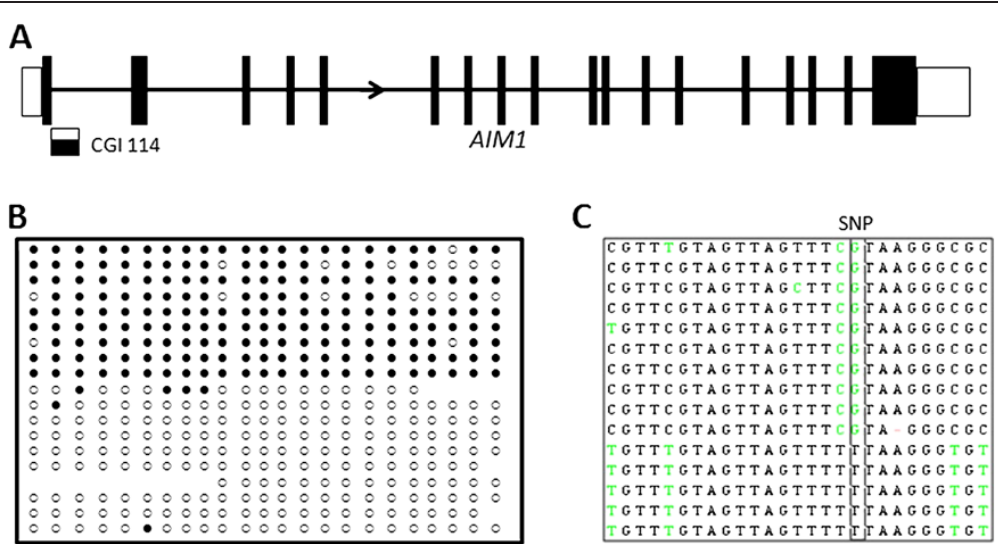

D

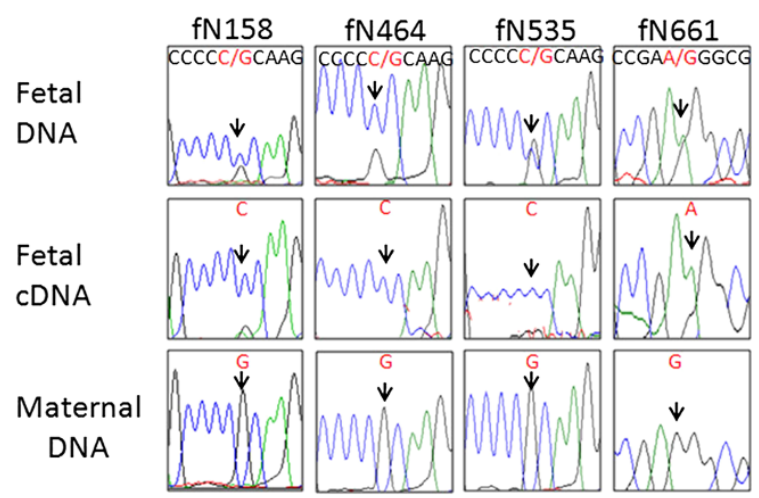

Figure 4 Methylation and expression analyses of AIM1 in human placenta. (A) CGI 114 is located at the junction of exon 1 and intron 1 of the long transcript of AIM1. (B) Bisulfite treatment and cloning confirmed about 50\% methylation in an allele specific manner in human placenta. (C) Sequencing of clones from the heterozygous individual fN158 showed that the maternal allele (G allele) was associated with methylated CpGs, while the paternal allele ( $T$ allele) was associated with unmethylated CpGs. (D) AlM1 was paternally expressed in humans. Arrow depicts the SNP location for each sample. In all four samples, the fetal DNA was heterozygous and the maternal DNA was homozygous. The fetal RNA showed monoallelic expression from the paternal allele.

The Chromosome 19 DMR is located at the promoter of the well-studied DNMT1 gene. DNMT1 selectively methylates hemi-methylated DNA, regulates tissuespecific methylation and is also essential for maintenance of progenitor cells in an undifferentiated state in somatic tissues [62]. It produces two transcripts, one expressed in somatic tissues (s-DNMT1) and the other expressed specifically in the oocyte (o-DNMT1). The promoter of $s-D N M T 1$ was shown to be monoallelically methylated specifically in the primate placenta and to be hypomethylated in other human tissues [63]. Novakovic et al. reported the monoallelic methylation in human placenta to be random, based on only one sample harboring a SNP (rs8112895) in the promoter of $s-D N M T 1$. In another recent report, an elegant screen for potential DMRs using diandric and digynic conceptuses also identified the same DMR at the s-DNMT1 locus, although again only one informative individual was analyzed to confirm monoallelic expression [64]. In our study, we confirmed monoallelic expression in eight individual samples, paternal allele-specific expression in four of these individuals, and maternal specific methylation in one sample with an informative SNP. The latter SNP is located in the CGI within the DNMT1 promoter (within exon 1, 428-bp away from the polymorphism used in the earlier report [63].

It is interesting to note that the promoter of $s$-DNMT1 has already been shown to be monoallelically methylated in the placenta of baboons and marmosets (Figure 4, [48]). Thus, it is likely that placental genomic imprinting of DNMT1 is maintained throughout the primate lineage. Since we lacked parent-offspring matched samples for our macaque tissues, we were unable to confirm the parental-allele specific expression of DNMT1 in this species.

It is also interesting that the promoter of $s-D N M T 1$ has been shown to be unmethylated in the mouse placenta (Figure 4 [48]). However, dynamic methylation changes have been observed upstream of the o-DNMT1 transcript during early mouse development [65]. No evidence for imprinting of murine Dnmt1 has emerged from genome-wide placenta specific imprinting studies 
A

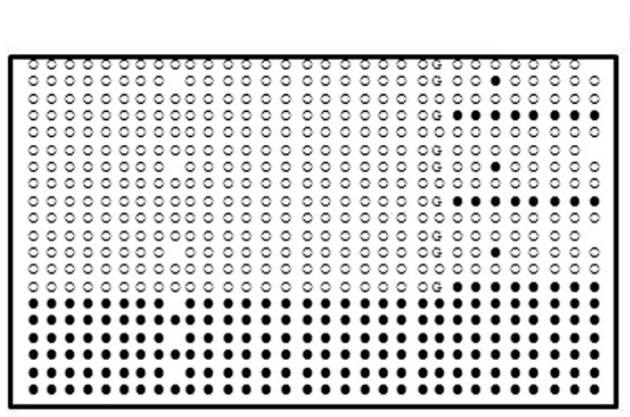

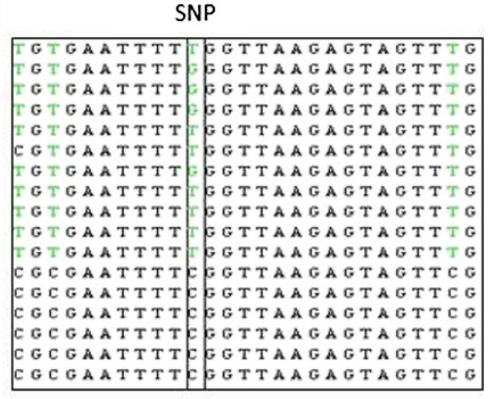

C

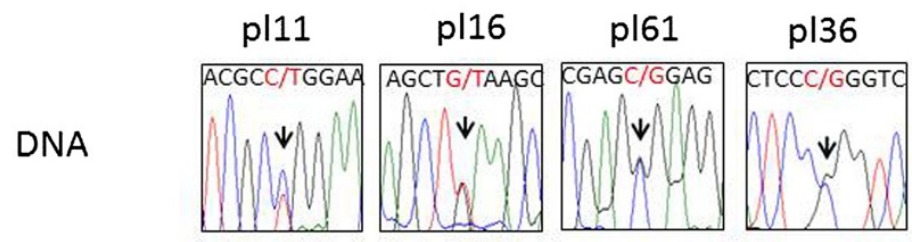

cDNA
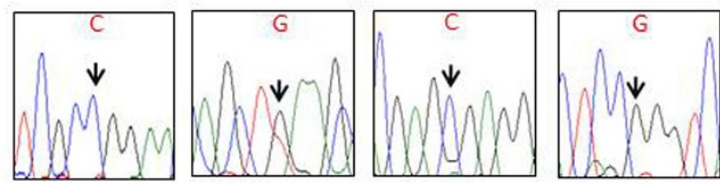

Figure 5 Methylation and expression analyses of AIM1 in macaque placenta. (A) The region homologous to the AIM1 DMR in the macaque exhibited approximately 34\% methylation. (B) Sequencing of clones from the heterozygous individual pl36 showed that the expressed allele (G allele) was associated with unmethylated CpGs, while the non-expressed allele (C allele) was partially methylated. (C) AIM1 was monoallelically expressed in the macaque. Arrow depicts the SNP location for each sample. In all four samples, the DNA was heterozygous and the RNA showed monoallelic expression.

in mice $[18,23]$. Thus, it appears that genomic imprinting of DNMT1 is specific to the primate placenta.

The function of the paternal allele-specific expression of DNMT1 in human placenta remains to be elucidated. Methylation of the $s-D N M T 1$ promoter may attenuate its transcription; this is coincident with global hypomethylation of the human placenta [63]. Moreover, s-DNMT1 expression attenuation has been reported to cause alterations in methylation at germline DMRs $[66,67]$. Thus, it is possible that reduction in $s$-DNMT1 level in the human placenta by genomic imprinting is linked to loss-of-imprinting observed at several loci in this tissue $[38,68]$.

AIM1 or Absent in Melanoma 1 is a non-lens member of the $\beta \gamma$-crystallin superfamily [69,70]. It was predicted to be a suppressor of malignant melanoma and NK-cell malignancies [71]. It was implicated in trophoblast differentiation in the placenta [72]. It has two alternative transcripts and both are highly expressed in the placenta.

The Chromosome 6 DMR (CpG 114) lies at the exon1-intron1 junction of the long transcript of gene AIM1, 460-bp downstream of the transcription start site. There is another CpG island (CpG 44) located 587-bp upstream of the transcription start site of this transcript. CpG 44 is unmethylated. Thus, CpG 114 potentially regulates the expression of the long transcript. The DMR and imprinted expression were found to be conserved in the macaque placenta, but not in the mouse placenta. It should be noted that in the macaque placenta the nonexpressed allele was partially methylated while in the human placenta the non-expressed allele was fully methylated. We suspect that this was due to maternal cell contamination in the macaque samples, since the macaque placenta is much thinner than the human placenta, making it difficult to isolate pure fetal cells.

It is interesting to speculate about the function of paternal allele-specific expression of the long transcript of AIM1. Since this transcript appears to be robustly expressed in the placenta [70], it is possible that its expression regulated by imprinting is functionally relevant in this tissue. IGF2R, one example of a maternally expressed imprinted gene is located on the same chromosome. However, it is unlikely that they belong to the same imprinted cluster since they are approximately $53 \mathrm{Mb}$ apart. Moreover, IGF2R exhibits polymorphic imprinting in humans [73].

Limited numbers of validated novel imprinted genes were discovered in previous genome-wide screens, raising the question whether most imprinted genes had been identified [36]. Despite evidence suggesting extensive loss of imprinting in the human placenta [74], our study as 


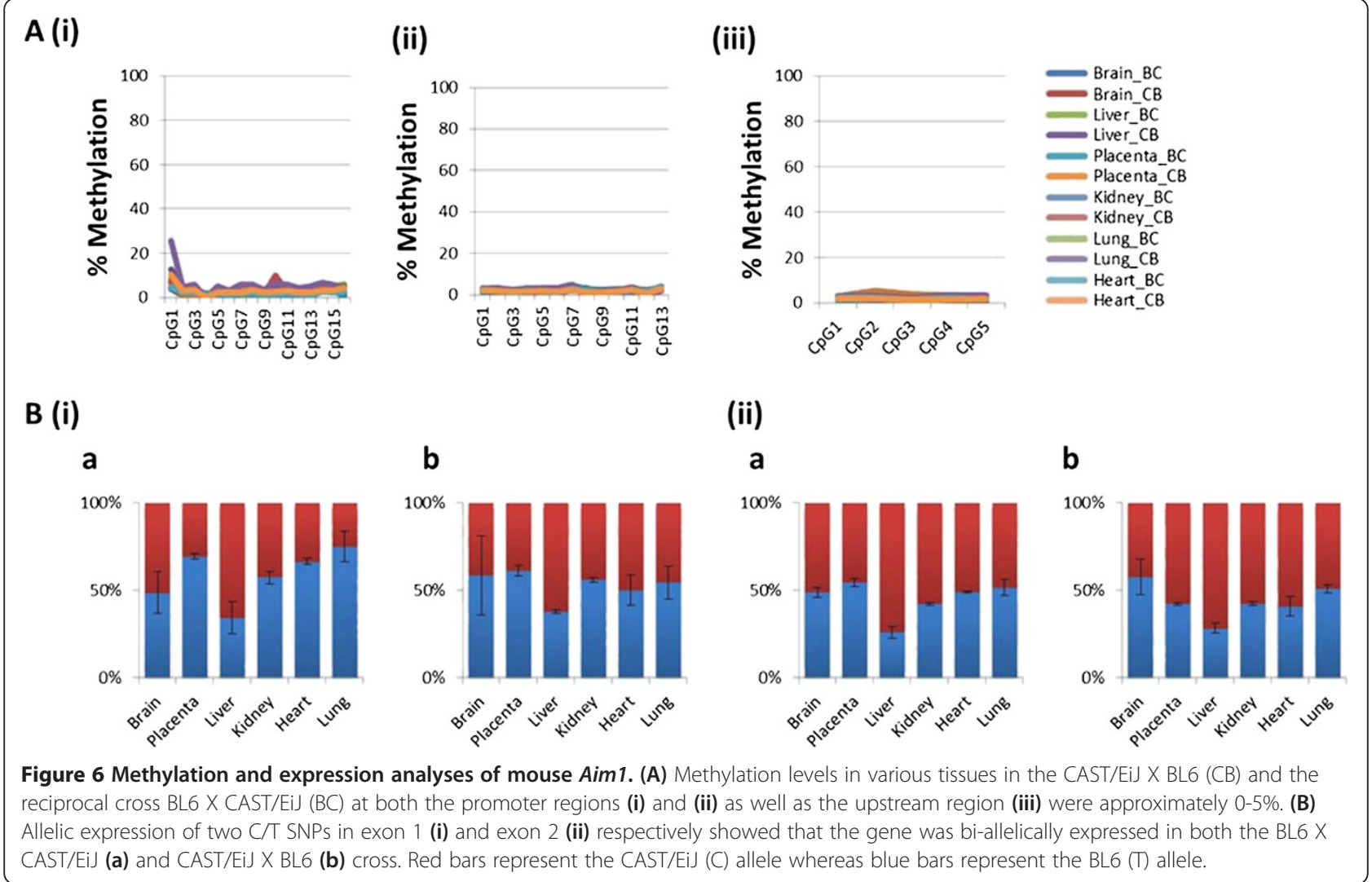

well as others [21,37] suggest that novel species and tissue-specific imprinted genes remain to be discovered [4]. The functional consequences of such imprinting events may be species, tissue, and even developmental stage specific. In this regard, the placenta may be a good tissue for studying genomic imprinting since it is both functionally important and evolutionarily under intense selective pressure.

However it is also clear from our data that while allele-specific DNA methylation may be prevalent, most of these epigenetically regulated regions are not associated with genomic imprinting. Out of the 28 potential DMRs analyzed, only two were shown to be imprinted DMRs. We confirmed the allele-specific methylation profile of 10 additional regions by bisulfite cloning and sequencing, even though these were not associated with monoallelic expression. Many of these potential DMRs are located in gene-bodies (Figure 2C and 2D). Some of these regions could contribute to processes like alternative splicing [75], or replication timing [76].

One limitation of our study is the use of RRBS rather than whole genome bisulfite sequencing for the discovery of imprinted genes. RRBS enriches for CpG-rich regions, particularly CpG islands. It is possible that this led to identification of only maternally methylated DMRs in our study, since all known paternally methylated gDMRs are in CpG-poor, inter-genic regions. Analysis of the ten partially methylated regions which were fully methylated in sperm and were potential paternal gDMRs did not yield any imprinted gene candidates. Whole genome bisulfite sequencing analyses would facilitate future discovery of

\section{Conclusions}

In conclusion, we have developed a method to study allele-specific methylation and associated genomic imprinting in the human placenta. Careful follow-up and validation of other partially methylated regions with high concordance will potentially reveal the functional role of methylation in these regions and may help identify more novel imprinted genes. The precise functions and mechanisms associated with placenta-specific imprinting of DNMT1 and AIM1 remain to be investigated. A more complete catalog of species-specific imprinted genes in the placenta will help our understanding of how genomic imprinting is associated with placental function, morphology and evolution.

\section{Methods}

Study participants and sample processing

Women with euploidy pregnancies who attended KK Women's and Children's Hospital, Singapore, were gDMRs in an unbiased manner. 
recruited. Informed consent was obtained under the ethics approval from the SingHealth CRIB Committee (IRB Reference: EC200903042, CIRB Reference: 2009/271/A). Maternal ethnicity was ascertained to be Chinese in four out of the five first trimester placenta cases and Asian for the remaining one. Amongst the four third trimester placenta cases, three were Chinese but the maternal ethnicity for one subject was not available.

Ten $\mathrm{mL}$ of peripheral blood from each subject was collected in EDTA tubes. The blood samples were centrifuged at $1,790 \mathrm{~g}$ for $10 \mathrm{~min}$ at $4^{\circ} \mathrm{C}$. After removing the supernatant plasma, the blood cells were transferred to a new microcentrifuge tube and centrifuged at 2,300 g for 5 minutes at room temperature to remove the residual plasma. The blood cells containing buffy coat were then collected and stored at $-80^{\circ} \mathrm{C}$. DNA was extracted from $200 \mu \mathrm{L}$ of blood cells using QIAamp DNA Blood Mini Kit (QIAGEN GmbH, Germany), according to manufacturer's instructions. DNA samples eluted with $50 \mu \mathrm{L}$ of DNase and RNase-free water (Sigma-Aldrich, St. Louis, MO, USA) were stored at $-80^{\circ} \mathrm{C}$.

First trimester villi samples were collected from leftover material obtained via chorionic villus sampling. The villi samples were washed extensively with diethylpyrocarbonate (DEPC)-treated water. Third trimester placental villi were collected from subjects after the normal babies were delivered. The fetal side of the placenta was washed with $1 \times \mathrm{PBS}$ before dissection. A small piece of placenta tissue $1 \mathrm{~cm}$ below the chorionic plate was dissected and maternal blood was thoroughly washed away with DEPCtreated water. These samples were stored at $-80^{\circ} \mathrm{C}$ immediately (for DNA extraction), or incubated with RNAlater (Applied Biosystems/Ambion, Carlsbad, CA, USA) overnight at $4^{\circ} \mathrm{C}$, and stored at $-80^{\circ} \mathrm{C}$ (for RNA extraction). DNA and RNA extractions were performed with QIAamp DNA Mini Kit (QIAGEN) and TRIZOL Reagent (Invitrogen, Carlsbad, CA, USA), respectively, according to manufacturer's instructions.

\section{Reduced representation bisulfite sequencing (RRBS) and $\mathrm{GA}$ analysis}

One to five microgram each of genomic DNA from the nine human placental samples was fragmented by restriction enzyme digestion using both $\mathrm{Taq}^{\alpha} \mathrm{I}$ and MspI (New England Biolabs, Ipswich, MA, USA), and was end-repaired, 3'-end-adenylated, and adapter-ligated using ChIP-Seq Sample Preparation Kit (Illumina, San Diego, CA, USA). Illumina's RRBS for Methylation Analysis protocol was followed, except that $10 \mu \mathrm{L}$ of the methylation adapter oligo was used and the ligation was performed for $15 \mathrm{~min}$ at $20^{\circ} \mathrm{C}$ in the adapter-ligation step. The gel-purified fragments were then bisulfite treated using the EZ DNA Methylation-Gold Kit (Zymo Research, Irvine, CA, USA), according to manufacturer's instructions. The converted DNA was PCR enriched, purified by gel electrophoresis, and the library was validated using Agilent 2100 Bioanalyzer (Agilent Technologies, Santa Clara, CA, USA). RRBS was performed on the Illumina Genome Analyzer IIx platform, as per manufacturer's instructions. The paired-end 36-base pair (bp) reads were filtered based on qseq score, then aligned to CGIs in the bisulfite-converted human genome (UCSC Build hg19, GRCh37, Feb 2009) using Bowtie [77]. The formula for computing bisulfite conversion rate was:

$$
\begin{aligned}
& \text { Bisulfite Conversion Percentage } \\
& =(\text { nonCpGC- }>\mathrm{T}) /(\text { nonCpGC- }>\mathrm{C}+\text { nonCpGC- }>\mathrm{T}) \\
& \quad * 100 \% .
\end{aligned}
$$

A bisulfite conversion rate above $99.3 \%$ was used as the cut-off.

The formula for computing the CpG methylation for the $\mathrm{CpG}$ sites was:

$$
\begin{aligned}
& \text { CpG Methylation Percentage } \\
& \qquad=(\text { CpGC- }>\text { C }) /(\text { CpGC- }>\text { C }+ \text { CpGC- }>\text { T }) * 100 \% .
\end{aligned}
$$

The \% CpG coverage achieved by RRBS is 3.3\%. Genomic regions with at least three CpGs covered at a minimum sequencing depth of 10 were considered. We covered $76.7 \%$ of all CGIs.

\section{Selection of regions with high concordance and partial methylation}

The average CGI methylation was obtained by averaging methylation percentage of all the CpGs in a defined CGI. This required that the CGI had at least $3 \mathrm{CpGs}$ with $10 \times$ or greater coverage. The CpGs were not necessarily within a limited interval but had to be inside the defined CGIs. CGIs with partial methylation ranging from $30 \%$ to $70 \%$ were selected. Those with high concordance $(>85 \%)$ were further short-listed. The concordance of adjacent CpGs in the same read was defined as the ratio of the number of identically methylated (or unmethylated) $\mathrm{CpG}$ pairs over the total number of $\mathrm{CpG}$ pairs. The average CGI concordance was obtained by averaging concordance of all the CpGs in the defined CGIs. The partially methylated CGIs with concordance $>85 \%$ were annotated based on whether they belonged to promoters, gene-bodies or inter-genic regions. The promoter of a gene was defined as $1000 \mathrm{bp}$ upstream to $500 \mathrm{bp}$ downstream of the transcription start site for this analysis.

The above analysis was carried out on the first and third trimester placentas separately. Comparing the lists of CGIs with partial methylation and high concordance 
from the first and third trimester placentas, there was an overlap of 495 CGIs shared between the two.

To assess the possibility of false positives in our dataset of partially methylated genomic regions, we analyzed 37 CGIs known to be unmethylated in first trimester placenta samples [78]. Nine of these 37 CGIs were not analyzed by the RRBS methodology used in this study. The remaining 28 CGIs were almost completely unmethylated in our data (average methylation 2.62\%, Additional file 9). None of these 28 CGIs were present amongst the candidate regions identified in our dataset, indicating a low false positive rate.

To shortlist CGIs that are potential gDMRs, MeDIP methylation data for three human spermatozoa samples was downloaded from http://www.ncbi.nlm.nih.gov/geo/ query/acc.cgi?acc=GSE25686 [58]. Overlapping CGIs between the two data sets were compared to identify regions that exhibited $100 \%$ methylation in the sperm but partial methylation with high concordance in the placental samples from our data.

\section{Imprinting analysis in human placental samples}

For assessing monoallelic expression status of the candidate imprinted genes, we first sequenced DNA of exons containing high frequency SNPs (based on dbSNP) for genes listed in Tables 2 and 3. Once heterozygous samples were identified, we tested whether the gene was monoallelically expressed.

Eighty one human placental tissues were chosen for imprint analysis. Thirty ng of purified genomic DNA was used for amplification with the HotStar Taq DNA Polymerase Kit (QIAGEN) with the addition of Q solution (primer sequences are listed in Additional file 7: Table S2). The thermocycling condition was $15 \mathrm{~min}$ at $95^{\circ} \mathrm{C}$ for heat activation, and $45 \mathrm{cycles}$ of $20 \mathrm{sec}$ at $94^{\circ} \mathrm{C}, 30 \mathrm{sec}$ at $60^{\circ} \mathrm{C}$ and $1 \mathrm{~min}$ at $72^{\circ} \mathrm{C}$, followed by a 3-min final extension at $72^{\circ} \mathrm{C}$. The amplicons were sequenced using BigDye Terminator v3.1 Cycle Sequencing Kit (Applied Biosystems). Reverse transcription was performed using gene-specific reverse primer (AIM1RT1 for AIM1) or oligo dT primer (for the rest of the genes, including DNMT1) and Superscript III (Invitrogen). The cDNA obtained was amplified using specific primers (listed in Additional file 7: Table S2) and the same thermocycling conditions as above. The PCR products were subsequently sequenced as above. To determine parent-of-origin expression, maternal blood DNA was also sequenced. An informative case was where the mother was homozygous and the fetus was heterozygous.

To increase throughput, Sequenom multiplex genotyping assays were performed on genes listed in Table 3. Sequenom Typer 4.0 (Sequenom, Inc., San Diego, CA, USA) was used to design four multiplex reactions for 30 SNPs within the 10 selected genes. Twenty eight sample sets (fetal placental DNA, buffy coat maternal DNA and fetal placental cDNA) were used at a concentration of $20 \mathrm{ng} / \mu \mathrm{L}$ in $5 \mu \mathrm{L}$ PCRs (as above) with $0.5 \mu \mathrm{M}$ primer concentration and thermocycling conditions of $15 \mathrm{~min}$ at $94{ }^{\circ} \mathrm{C}$ for heat activation, and 45 cycles of $20 \mathrm{sec}$ at $94^{\circ} \mathrm{C}, 30 \mathrm{sec}$ at $56^{\circ} \mathrm{C}$ and $1 \mathrm{~min}$ at $72^{\circ} \mathrm{C}$, followed by a 3 -min final extension at $72^{\circ} \mathrm{C}$. The PCR products were treated with Shrimp Alkaline Phosphatase for 40 minutes at $37^{\circ} \mathrm{C}$ followed by 5 minutes at $85^{\circ} \mathrm{C}$. The extension reaction was performed as per the manufacturer's instructions, with a $9 \mu \mathrm{M}$ extension primer concentration. The sample clean-up, spotting on the chip and laser firing were done as per the manufacturer's instructions and the data was analyzed using the Typer software.

\section{Methylation analysis by cloning and bisulfite sequencing} Bisulfite conversion with $1 \mu \mathrm{g}$ of each genomic DNA sample was performed using the EZ DNA MethylationGold Kit (Zymo Research), according to manufacturer's instructions. One twenty-fifth of converted DNA was used for each PCR (primers listed in Additional file 7: Table S1) with HotStar Taq DNA Polymerase Kit (QIAGEN). The thermocycling condition was $15 \mathrm{~min}$ at $95^{\circ} \mathrm{C}$ for heat activation, and 50 cycles of $20 \mathrm{sec}$ at $94^{\circ} \mathrm{C}$, $30 \mathrm{sec}$ at $62^{\circ} \mathrm{C}$ and $1 \mathrm{~min}$ at $72^{\circ} \mathrm{C}$, followed by a 3-min final extension at $72^{\circ} \mathrm{C}$. The PCR product was TAcloned into pGEM-T Easy vector (Promega, Madison, WI, USA), and the positive clones with inserts were then subjected to PCR amplification using SP6 and T7 primer set from the vector. Sequencing reaction was performed using BigDye Terminator v3.1 Cycle Sequencing Kit (Applied Biosystems).

\section{Methylation and imprinting analyses of AIM1 in macaques} Eleven Cynomolgus macaque (Macaca fascicularis) neonatal and placental tissues were collected from the Vietnam Primate Breeding and Development Centre. All animal procedures were approved by Nafovanny, subsidiary of the Ministry of Forestry, Vietnam, and performed in accordance with the guidelines set by the national advisory committee for laboratory animal research (NACLAR) of Singapore. All harvested samples were stored at $-80^{\circ} \mathrm{C}$ until analyses.

A CGI homologous to human CpG 114 was identified by BLAT at chr4:102,561,846-102,563,066 on the macaque (Macaca mulatta) genome available on the UCSC genome browser. DNA was isolated, bisulfite treated, amplified (primers listed in Additional file 7: Table S1), cloned into TA vector and sequenced as above.

For imprinting analysis, selective genomic regions were amplified from macaque AIM1 to analyze polymorphisms located at chr4:102,561,811-102,619,896 and Chr4:102,562,698-102,562,816 (primers listed in Additional file 7: Table S2, same thermocycling conditions as above but with annealing temperature $58^{\circ} \mathrm{C}$ ) and sequenced. The 
expression of the polymorphisms was then analyzed by extracting RNA from placenta of the informative individuals and reverse transcribing (using gene-specific RT primer MacaqueAIM1RT1 or RT2 as appropriate), amplifying with primers listed in Additional file 7: Table S2 and the same thermocycling conditions, and finally sequencing as above.

Additional tissues (liver, biceps, kidney, heart, lungs and pancreas) were available for two of the informative individuals, and these were also subject to methylation analysis at the DMR region.

\section{Imprinting analysis in mice}

Mouse Aim 1 is located at chr10:43670113-43724652. It is reasonably well-conserved with human AIM1, even though it is in reverse orientation unlike its human counterpart. A UCSC-defined CGI is annotated only at its promoter region (chr10:43723295-43724261), but an upstream region at chr10:43,725,821-43,726,151 with elevated H3K4me3 binding could also potentially serve as an alternate promoter. Thus, two bisulfitepyrosequencing assays were designed to analyze the methylation levels at the promoter CGI as well as one assay for the upstream region (primers listed in Additional file 7: Table S1). DNA from six tissues collected from two reciprocal hybrid (BL6 $\times$ CAST/EiJ) embryos at E16.5 stage were bisulfite treated and subject to quantitative CpG pyrosequencing analysis (as per manufacturer's instructions).

In order to analyze imprinting at this locus, the CAST/EiJ inbred strain crossed with the BL6 which contains polymorphisms in exon 1 and exon 2 of Aim 1 were used. RNA was extracted from the above mentioned hybrid embryo tissue material and cDNA was generated. Quantitative real-time PCR was performed to judge the relative levels of expression in the different tissues (brain, placenta, liver, kidney, heart, lung) using primers listed in Additional file 7: Table S2. The resultant PCR product was pyrosequenced (as per manufacturer's instructions) in order to quantify the relative proportion of each parental allele for the SNP rs46531577 at chr10:43723761 (within exon 1) and SNP rs29356879 at chr10:43717117 (within exon 2).

\section{Additional files}

Additional file 1: Figure S1. Methylation Analysis of Mouse gDMRs in Human Placental Tissue. Bisulfite cloning and sequencing showed that the promoters of imprinted genes INPP5Fv2 (A) and MCTS2 (B) were methylated in an allele-specific manner in human placental tissue.

Additional file 2: Lists the allele-specific methylated regions in human placenta.

Additional file 3: Figure S3. Confirmation of Allele-specific Expression and Methylation of DNMT1 and AIM1. (A) fN165 expressed the "T" allele in exon 1 of DNMT1, reciprocal of the " $\mathrm{C}$ " allele expressed at the " $\mathrm{C} / \mathrm{T}^{\text {" locus of }}$
fN158 shown in Figure 3D. Similarly, fN468 expressed the " $G$ " allele in exon 1 of $A / M 1$, reciprocal of the "A" allele expressed at the "A/G" locus of fN661 shown in Figure 4D. (B) fN134 was polymorphic within the DNMT1 DMR; the $\mathrm{G}$ allele was unmethylated whereas the A allele was methylated. (C) fN155 harbored a SNP in the AIM1 DMR; the G/maternal allele was associated with methylated clones whereas the T/paternal allele was associated with unmethylated clones. (D) mN158 was non-polymorphic within the AIM1 locus and still exhibited an allele-specific methylation profile, indicating that the methylation pattern was not a SNP effect.

Additional file 4: Figure S2. AIM1 Transcripts and Expression Analysis from Different Exons. Human AIM1 was mono-allelically expressed from exon 1 of Transcript 1 (an asterisk indicates the location of the SNP) but bi-allelic expression was observed by analyzing SNPs in the last exon (which overlaps with Transcript 2, double asterisk indicates the location of the SNP). The transcript information has been obtained from the Ensembl Genome Browser.

Additional file 5: Figure S4. Methylation analysis of the AIM1 DMR in Additional Macaque Tissues. All tissues other than placenta: liver (A), biceps $(B)$, kidney $(C)$, lung $(D)$, heart $(E)$ and pancreas $(F)$ were found to be unmethylated at the DMR locus.

Additional file 6: Figure S5. Expression Analysis of AIM1 in Macaque Tissues. (A) qPCR for AIM1 in different macaque tissues of one individual (Macaque 11) showed that it is expressed only in placenta, heart and kidney tissues. (B) Macaque AIM1 is bi-allelically expressed in the heart and kidney tissue of the same individual. Arrow depicts the genomic location of a $C / T$ polymorphism that was still apparent in the CDNA.

Additional file 7: Table S1. Primers Used for Methylation Analysis of DNMT1 and AIM1. Table S2. Primers Used for Analysis of Imprint Status of DNMT1 and AIM1. Table S3. Methylation percentage for individual CpG sites in various tissues in the mouse for Aim 1 promoter and upstream region.

Additional file 8: Figure S6. Expression Analysis of Aim1 in Mouse Tissues. qPCR for Aim 1 in a panel of mouse tissues showed that it is expressed only in placenta, heart and kidney tissues from both Exon 1 (A) and Exon 2 (B). Blue bars represent the BL6 X CAST/EiJ cross allele whereas red bars represent the CAST/EiJ X BL6 reciprocal cross.

Additional file 9: Lists the loci used to assess false positives. The RRBS and RNA-seq data have been submitted to NCBI Gene Expression Omnibus under accession no. GSE40955.

\section{Abbreviations}

CGI: CPG island; DMR: Differentially methylated region; RRBS: Reduced representation bisulfite sequencing; MeDIP: Methylated DNA immunoprecipitation; DNMT1: DNA Methyl Transferase 1; AlM1: Absent in Melanoma 1.

\section{Competing interests}

The authors declare that they have no competing interests.

\section{Authors' contributions}

$\mathrm{RD}$ performed the imprinting analysis experiments in humans and macaques and wrote the manuscript. YKL and YCL analyzed the RRBS data. RS performed the mouse experiments. SJ performed the RRBS experiments and aided human sample collection. PYN ran the machines for RRBS. XML collected the human placenta samples. KC collected the macaque samples. GSKY facilitated human sample collection from the clinic. AFS helped design the mouse experiments and aided preparation of the manuscript. DC helped with conceptualization, experimental design and manuscript preparation. All authors read and approved the final manuscript.

\section{Acknowledgements}

The authors thank the doctors and patients at KK Women's and Children's hospital, Singapore for their participation in this study. We are also grateful to past members of the Ferguson-Smith Laboratory when at SICS (Dr. Aileen Lim, Dr. Clara Cheong and Rachel Chew) for kindly providing the macaque samples.

This work was supported by an A*STAR grant to Chunming Ding. CD/GSHY is also supported by a Bench to Bedside grant (09/1/50/19/622) from 
Biomedical Research Council and National Medical Research Council (BMRCNMRC). RS is supported by a UK Biotechnology and Biological Sciences Research Council grant to A.C.F-S.

\section{Author details}

${ }^{1}$ Singapore Institute for Clinical Sciences, Agency for Science, Technology and Research (A*STAR), Singapore, Singapore. ${ }^{2}$ Department of Physiology, Development \& Neuroscience, University of Cambridge, Cambridge, UK. ${ }^{3}$ Department of Maternal Fetal Medicine, K.K. Women's and Children's Hospital, Singapore, Singapore.

Received: 30 January 2013 Accepted: 25 September 2013

Published: 5 October 2013

\section{References}

1. Bartolomei MS, Ferguson-Smith AC: Mammalian genomic imprinting. Cold Spring Harb Perspect Biol 2011, 3(7):1-15.

2. Reik W, Walter J: Evolution of imprinting mechanisms: the battle of the sexes begins in the zygote. Nat Genet 2001, 27(3):255-256.

3. Okamura K, Ito T: Lessons from comparative analysis of species-specific imprinted genes. Cytogenet Genome Res 2006, 113(1-4):159-164.

4. Prickett AR, Oakey RJ: A survey of tissue-specific genomic imprinting in mammals. Mol Genet Genomics 2012, 287(8):621-630

5. Das R, Hampton DD, Jirtle RL: Imprinting evolution and human health. Mamm Genome 2009, 20(9-10):563-572.

6. John RM, Lefebvre L: Developmental regulation of somatic imprints. Differentiation 2011, 81(5):270-280.

7. Lewis A, Mitsuya K, Umlauf D, Smith P, Dean W, Walter J, Higgins M, Feil R, Reik W: Imprinting on distal chromosome 7 in the placenta involves repressive histone methylation independent of DNA methylation. Nat Genet 2004, 36(12):1291-1295.

8. Morcos L, Ge B, Koka V, Lam KC, Pokholok DK, Gunderson KL, Montpetit A, Verlaan DJ, Pastinen T: Genome-wide assessment of imprinted expression in human cells. Genome Biol 2011, 12(3):R25

9. Brideau CM, Eilertson KE, Hagarman JA, Bustamante CD, Soloway PD: Successful computational prediction of novel imprinted genes from epigenomic features. Mol Cell Biol 2010, 30(13):3357-3370.

10. Luedi PP, Dietrich FS, Weidman JR, Bosko JM, Jirtle RL, Hartemink AJ: Computational and experimental identification of novel human imprinted genes. Genome Res 2007, 17(12):1723-1730.

11. Strichman-Almashanu LZ, Lee RS, Onyango PO, Perlman E, Flam F, Frieman MB, Feinberg AP: A genome-wide screen for normally methylated human CpG islands that can identify novel imprinted genes. Genome Res 2002, 12(4):543-554.

12. Ishino F, Kuroiwa Y, Miyoshi N, Kobayashi S, Kohda T, Kaneko-Ishino T: Subtraction-hybridization method for the identification of imprinted genes. Methods Mol Biol 2001, 181:101-112.

13. Pollard KS, Serre D, Wang X, Tao H, Grundberg E, Hudson TJ, Clark AG, Frazer K: A genome-wide approach to identifying novel-imprinted genes. Hum Genet 2008, 122(6):625-634

14. Schulz R, Menheniott TR, Woodfine K, Wood AJ, Choi JD, Oakey RJ: Chromosome-wide identification of novel imprinted genes using microarrays and uniparental disomies. Nucleic Acids Res 2006, 34(12):e88.

15. Smith RJ, Dean W, Konfortova G, Kelsey G: Identification of novel imprinted genes in a genome-wide screen for maternal methylation. Genome Res 2003, 13(4):558-569.

16. Wen $B$, Wu H, Bjornsson $H$, Green RD, Irizarry R, Feinberg AP: Overlapping euchromatin/heterochromatin- associated marks are enriched in imprinted gene regions and predict allele-specific modification. Genome Res 2008, 18(11):1806-1813

17. Choufani S, Shapiro JS, Susiarjo M, Butcher DT, Grafodatskaya D, Lou Y, Ferreira JC, Pinto D, Scherer SW, Shaffer LG, et al: A novel approach identifies new differentially methylated regions (DMRs) associated with imprinted genes. Genome Res 2011, 21(3):465-476.

18. Okae H, Hiura H, Nishida Y, Funayama R, Tanaka S, Chiba H, Yaegashi N, Nakayama K, Sasaki H, Arima T: Re-investigation and RNA sequencingbased identification of genes with placenta-specific imprinted expression. Hum Mol Genet 2011, 2011:2011.

19. Nakabayashi K, Trujillo AM, Tayama C, Camprubi C, Yoshida W, Lapunzina P, Sanchez A, Soejima H, Aburatani H, Nagae G, et al: Methylation screening of reciprocal genome-wide UPDs identifies novel human-specific imprinted genes. Hum Mol Genet 2011, 20(16):3188-3197.
20. Babak T, Deveale B, Armour C, Raymond C, Cleary MA, van der Kooy D, Johnson JM, Lim LP: Global survey of genomic imprinting by transcriptome sequencing. Curr Biol 2008, 18(22):1735-1741.

21. Barbaux S, Gascoin-Lachambre G, Buffat C, Monnier P, Mondon F, Tonanny MB, Pinard A, Auer J, Bessieres B, Barlier A, et al: A genome-wide approach reveals novel imprinted genes expressed in the human placenta. Epigenetic 2012, 7(9):1079-1090.

22. Proudhon C, Duffie R, Ajjan S, Cowley M, Iranzo J, Carbajosa G, Saadeh H, Holland ML, Oakey RJ, Rakyan VK, et al: Protection against de novo methylation is instrumental in maintaining parent-of-origin methylation inherited from the gametes. Mol Cell 2012, 47(6):909-920.

23. Wang $X$, Soloway PD, Clark AG: A survey for novel imprinted genes in the mouse placenta by mRNA-seq. Genetics 2011, 189(1):109-122.

24. Gregg C, Zhang J, Butler JE, Haig D, Dulac C: Sex-specific parent-of-origin allelic expression in the mouse brain. Science 2010, 329(5992):682-685.

25. Gregg C, Zhang J, Weissbourd B, Luo S, Schroth GP, Haig D, Dulac C: Highresolution analysis of parent-of-origin allelic expression in the mouse brain. Science 2010, 329(5992):643-648.

26. DeVeale B, van der Kooy D, Babak T: Critical evaluation of imprinted gene expression by RNA-Seq: a new perspective. PLoS Genet 2012, 8(3):e1002600.

27. Xie W, Barr CL, Kim A, Yue F, Lee AY, Eubanks J, Dempster EL, Ren B: Baseresolution analyses of sequence and parent-of-origin dependent DNA methylation in the mouse genome. Cell 2012, 148(4):816-831.

28. Bressan FF, De Bem TH, Perecin F, Lopes FL, Ambrosio CE, Meirelles FV, Miglino MA: Unearthing the roles of imprinted genes in the placenta. Placenta 2009, 30(10):823-834.

29. Constancia M, Kelsey G, Reik W: Resourceful imprinting. Nature 2004, 432(7013):53-57.

30. Fowden AL, Sibley C, Reik W, Constancia M: Imprinted genes, placental development and fetal growth. Horm Res 2006, 65(Suppl 3):50-58.

31. Frost JM, Moore GE: The importance of imprinting in the human placenta. PLoS Genet 2010, 6(7):e1001015.

32. Goshen R, Ben-Rafael Z, Gonik B, Lustig O, Tannos V, de Groot N, Hochberg AA: The role of genomic imprinting in implantation. Fertil Steril 1994, 62(5):903-910.

33. Pliushch G, Schneider E, Weise D, El Hajj N, Tresch A, Seidmann L, Coerdt W, Muller AM, Zechner $U$, Haaf T: Extreme methylation values of imprinted genes in human abortions and stillbirths. Am J Pathol 2010, 176(3):1084-1090.

34. Uuskula L, Rull K, Nagirnaja L, Laan M: Methylation allelic polymorphism (MAP) in chorionic gonadotropin beta5 (CGB5) and its association with pregnancy success. J Clin Endocrinol Metab 2011, 96(1):E199-E207.

35. Yuen RK, Avila L, Penaherrera MS, von Dadelszen P, Lefebvre L, Kobor MS, Robinson WP: Human placental-specific epipolymorphism and its association with adverse pregnancy outcomes. PLoS One 2009, 4(10):e7389.

36. Daelemans C, Ritchie ME, Smits G, Abu-Amero S, Sudbery IM, Forrest MS Campino S, Clark TG, Stanier P, Kwiatkowski D, et al: High-throughput analysis of candidate imprinted genes and allele-specific gene expression in the human term placenta. BMC Genet 2010, 11:25.

37. Noguer-Dance M, Abu-Amero S, Al-Khtib M, Lefevre A, Coullin P, Moore GE, Cavaille J: The primate-specific microRNA gene cluster (C19MC) is imprinted in the placenta. Hum Mol Genet 2010, 19(18):3566-3582.

38. Monk D, Arnaud P, Apostolidou S, Hills FA, Kelsey G, Stanier P, Feil R, Moore GE: Limited evolutionary conservation of imprinting in the human placenta. Proc Natl Acad Sci U S A 2006, 103(17):6623-6628.

39. Lefebvre L: The placental imprintome and imprinted gene function in the trophoblast glycogen cell lineage. Reprod Biomed Online 2012, 25(1):44-57.

40. Hudson QJ, Seidl Cl, Kulinski TM, Huang R, Warczok KE, Bittner R, Bartolomei MS, Barlow DP: Extra-embryonic-specific imprinted expression is restricted to defined lineages in the post-implantation embryo. Dev Biol 2011, 353(2):420-431.

41. Proudhon C, Bourc'his D: Identification and resolution of artifacts in the interpretation of imprinted gene expression. Brief Funct Genomics 2010, 9(5-6):374-384.

42. Arima T, Yamasaki K, John RM, Kato K, Sakumi K, Nakabeppu Y, Wake N, Kono T: The human HYMAI/PLAGL1 differentially methylated region acts as an imprint control region in mice. Genomics 2006, 88(5):650-658.

43. Riesewijk AM, Xu YQ, Schepens MT, Mariman EM, Polychronakos C, Ropers $\mathrm{HH}$, Kalscheuer VM: Absence of an obvious molecular imprinting mechanism in a human fetus with monoallelic IGF2R expression. Biochem Biophys Res Commun 1998, 245(1):272-277.

44. Arnaud P, Monk D, Hitchins M, Gordon E, Dean W, Beechey CV, Peters J, Craigen W, Preece $M$, Stanier $P$, et al: Conserved methylation imprints in 
the human and mouse GRB10 genes with divergent allelic expression suggests differential reading of the same mark. Hum Mol Genet 2003, 12(9):1005-1019.

45. Monk D, Wagschal A, Arnaud P, Muller PS, Parker-Katiraee L, Bourc'his D, Scherer SW, Feil R, Stanier P, Moore GE: Comparative analysis of human chromosome 7q21 and mouse proximal chromosome 6 reveals a placental-specific imprinted gene, TFPI2/Tfpi2, which requires EHMT2 and EED for allelic-silencing. Genome Res 2008, 18(8):1270-1281.

46. Riesewijk AM, Hu L, Schulz U, Tariverdian G, Hoglund P, Kere J, Ropers HH, Kalscheuer VM: Monoallelic expression of human PEG1/MEST is paralleled by parent-specific methylation in fetuses. Genomics 1997, 42(2):236-244.

47. Kerjean A, Dupont JM, Vasseur C, Le Tessier D, Cuisset L, Paldi A, Jouannet $P$, Jeanpierre M: Establishment of the paternal methylation imprint of the human $\mathrm{H} 19$ and MEST/PEG1 genes during spermatogenesis. Hum Mol Genet 2000, 9(14):2183-2187.

48. Du M, Beatty LG, Zhou W, Lew J, Schoenherr C, Weksberg R, Sadowski PD: Insulator and silencer sequences in the imprinted region of human chromosome 11p15.5. Hum Mol Genet 2003, 12(15):1927-1939.

49. Kagami M, Sekita Y, Nishimura G, Irie M, Kato F, Okada M, Yamamori S, Kishimoto H, Nakayama M, Tanaka Y, et al: Deletions and epimutations affecting the human 14q32.2 imprinted region in individuals with paternal and maternal upd(14)-like phenotypes. Nat Genet 2008, 40(2):237-242.

50. El-Maarri O, Buiting K, Peery EG, Kroisel PM, Balaban B, Wagner K, Urman B, Heyd J, Lich C, Brannan Cl, et al: Maternal methylation imprints on human chromosome 15 are established during or after fertilization. Nat Genet 2001, 27(3):341-344

51. Huang JM, Kim J: DNA methylation analysis of the mammalian PEG3 imprinted domain. Gene 2009, 442(1-2):18-25.

52. Evans HK, Wylie AA, Murphy SK, Jirtle RL: The neuronatin gene resides in a "micro-imprinted" domain on human chromosome 20q11.2. Genomics 2001, 77(1-2):99-104.

53. Li J, Bench AJ, Vassiliou GS, Fourouclas N, Ferguson-Smith AC, Green AR: Imprinting of the human L3MBTL gene, a polycomb family member located in a region of chromosome 20 deleted in human myeloid malignancies. Proc Natl Acad Sci USA 2004, 101(19):7341-7346.

54. Liu J, Litman D, Rosenberg MJ, Yu S, Biesecker LG, Weinstein LS: A GNAS1 imprinting defect in pseudohypoparathyroidism type IB. J Clin Invest 2000, 106(9):1167-1174.

55. Liu J, Nealon JG, Weinstein LS: Distinct patterns of abnormal GNAS imprinting in familial and sporadic pseudohypoparathyroidism type IB. Hum Mol Genet 2005, 14(1):95-102.

56. Kanber D, Berulava T, Ammerpohl O, Mitter D, Richter J, Siebert R, Horsthemke $B$, Lohmann D, Buiting $K$ : The human retinoblastoma gene is imprinted. PLoS Genet 2009, 5(12):e1000790.

57. Monk D, Arnaud P, Frost JM, Wood AJ, Cowley M, Martin-Trujillo A, Guillaumet-Adkins A, Iglesias Platas I, Camprubi C, Bourc'his D, et al: Human imprinted retrogenes exhibit non-canonical imprint chromatin signatures and reside in non-imprinted host genes. Nucleic Acids Res 2011, 39(11):4577-4586.

58. Down TA, Rakyan VK, Turner DJ, Flicek P, Li H, Kulesha E, Graf S, Johnson N, Herrero J, Tomazou EM, et al: A Bayesian deconvolution strategy for immunoprecipitation-based DNA methylome analysis. Nat Biotechnol 2008, 26(7):779-785.

59. Mikeska T, Candiloro IL, Dobrovic A: The implications of heterogeneous DNA methylation for the accurate quantification of methylation. Epigenomics 2010, 2(4):561-573.

60. Fang F, Hodges E, Molaro A, Dean M, Hannon GJ, Smith AD: Genomic landscape of human allele-specific DNA methylation. Proc Natl Acad Sci U S A 2012, 109(19):7332-7337.

61. Peng $Q$, Ecker JR: Detection of allele-specific methylation through a generalized heterogeneous epigenome model. Bioinformatics 2012, 28(12):i163-i171.

62. Sen GL, Reuter JA, Webster DE, Zhu L, Khavari PA: DNMT1 maintains progenitor function in self-renewing somatic tissue. Nature 2010, 463(7280):563-567.

63. Novakovic B, Wong NC, Sibson M, Ng HK, Morley R, Manuelpillai U, Down T, Rakyan VK, Beck S, Hiendleder S, et al: DNA methylation-mediated downregulation of DNA methyltransferase-1 (DNMT1) is coincident with, but not essential for, global hypomethylation in human placenta. J Biol Chem 2010, 285(13):9583-9593.
64. Yuen RK, Jiang R, Penaherrera MS, McFadden DE, Robinson WP: Genomewide mapping of imprinted differentially methylated regions by DNA methylation profiling of human placentas from triploidies. Epigenetics Chromatin 2011, 4(1):10.

65. Ko YG, Nishino K, Hattori N, Arai Y, Tanaka S, Shiota K: Stage-by-stage change in DNA methylation status of Dnmt1 locus during mouse early development. J Biol Chem 2005, 280(10):9627-9634.

66. Borowczyk E, Mohan KN, D'Aiuto L, Cirio MC, Chaillet JR: Identification of a region of the DNMT1 methyltransferase that regulates the maintenance of genomic imprints. Proc Natl Acad Sci U S A 2009, 106(49):20806-20811.

67. Kurihara Y, Kawamura Y, Uchijima Y, Amamo T, Kobayashi H, Asano T, Kurihara $\mathrm{H}$ : Maintenance of genomic methylation patterns during preimplantation development requires the somatic form of DNA methyltransferase 1. Dev Biol 2008, 313(1):335-346.

68. Novakovic B, Saffery R: The ever growing complexity of placental epigenetics - role in adverse pregnancy outcomes and fetal programming. Placenta 2012, 33(12):959-970.

69. Aravind P, Wistow G, Sharma Y, Sankaranarayanan R: Exploring the limits of sequence and structure in a variant betagamma-crystallin domain of the protein absent in melanoma-1 (AIM1). J Mol Biol 2008, 381(3):509-518.

70. Ray ME, Wistow G, Su YA, Meltzer PS, Trent JM: AIM1, a novel non-lens member of the betagamma-crystallin superfamily, is associated with the control of tumorigenicity in human malignant melanoma. Proc Natl Acad Sci U S A 1997, 94(7):3229-3234.

71. Iqbal J, Kucuk C, Deleeuw RJ, Srivastava G, Tam W, Geng H, Klinkebiel D, Christman JK, Patel K, Cao K, et al: Genomic analyses reveal global functional alterations that promote tumor growth and novel tumor suppressor genes in natural killer-cell malignancies. Leukemia 2009, 23(6):1139-1151.

72. Handwerger $S$, Aronow B: Dynamic changes in gene expression during human trophoblast differentiation. Recent Prog Horm Res 2003, 58:263-281.

73. Killian JK, Nolan CM, Wylie AA, Li T, Vu TH, Hoffman AR, Jirtle RL: Divergent evolution in M6P/IGF2R imprinting from the Jurassic to the Quaternary. Hum Mol Genet 2001, 10(17):1721-1728.

74. Lambertini L, Diplas Al, Lee MJ, Sperling R, Chen J, Wetmur J: A sensitive functional assay reveals frequent loss of genomic imprinting in human placenta. Epigenetics 2008, 3(5):261-269.

75. Shukla S, Kavak E, Gregory M, Imashimizu M, Shutinoski B, Kashlev M Oberdoerffer P, Sandberg R, Oberdoerffer S: CTCF-promoted RNA polymerase II pausing links DNA methylation to splicing. Nature 2011, 479(7371):74-79.

76. Aran D, Toperoff G, Rosenberg M, Hellman A: Replication timing-related and gene body-specific methylation of active human genes. Hum Mol Genet 2011, 20(4):670-680.

77. Langmead B, Trapnell C, Pop M, Salzberg SL: Ultrafast and memoryefficient alignment of short DNA sequences to the human genome. Genome Biol 2009, 10(3):R25.

78. Chim SS, Jin S, Lee TY, Lun FM, Lee WS, Chan LY, Jin Y, Yang N, Tong YK, Leung TY, et al: Systematic search for placental DNA-methylation markers on chromosome 21: toward a maternal plasma-based epigenetic test for fetal trisomy 21. Clin Chem 2008, 54(3):500-511.

doi:10.1186/1471-2164-14-685

Cite this article as: Das et al:: DNMT1 and AIM1 Imprinting in human placenta revealed through a genome-wide screen for allele-specific DNA methylation. BMC Genomics 2013 14:685. 